\title{
Wave trains consisting of abruptly changing frequencies
}

\author{
WILLIAM J. MUNDL \\ Concordia University, Montreal, Quebec H3G 1M8, Canada
}

\begin{abstract}
Waveforms that contain points of abrupt transition from one frequency to another are useful in studies of visual perception. When applied to the Z-axis of an oscilloscope, such waveforms create vertical bars, which generate a wide variety of patterns, as required.
\end{abstract}

Waveform patterns containing abrupt transitions in frequencies and amplitudes are a valuable tool in studies of visual perception. Generally, such waveforms are applied to the $\mathrm{Z}$-axis of a cathode-ray oscilloscope (CRO), forming a pattern of vertical bars. To produce these patterns, a triangular waveform of sufficiently high frequency must be applied to the vertical input of the CRO. Choosing an appropriate internal sweep setting will then produce an even illumination of the entire screen. Intensity modulation at the $Z$-axis creates the desired pattem of vertical bars.

The waveform pattern is generated by an Intersil 8038 function generator, an integrated circuit device that was found to be extremely stable in regard to frequency and amplitude output. With appropriate control circuitry, the desired frequencies and durations of the waveform bursts can be satisfactorily produced. The described circuit arrangement provides for the generation of two adjustable frequencies and two adjustable time slots. This, in turn, creates a three-part waveform pattern on the CRO screen, consisting of Frequency $f_{1}$ over Time Window 1, Frequency $f_{2}$ over Time Window 2 , and then switching back to $f_{1}$ for the remaining sweep time.

\section{CIRCUIT DESCRIPTION}

A wave train is initiated by a positive pulse of $4 \mathrm{~V}$ minimum, derived from the CRO's time base. This pulse discharges the frequency-determining capacitor at Pin 10 of the function generator, ensuring that a wave train always begins at zero amplitude. Also triggered are multivibrators MV 1 and MV 2. With the timing components shown in Figure 1, the MVs generate

The author thanks Steven Stober for suggesting the usefulness of the described apparatus in research of visual perception and for his practical evaluation of the instrument. timing pulses of approximately .3 and $.6 \mathrm{msec}$, respectively. The end of the timing pulse produced by MV 1 switches the flip-flop (F-F) to "set," producing a high output at $\mathrm{Q}$. The end of the timing pulse produced by MV 2 resets the F-F. The two states of the F-F control the two frequencies, $f_{1}$ and $f_{2}$, appearing at the output of the 8038. The frequencies are adjustable with the two 5 -Kohm potentiometers. The 741 operational amplifier at the control input of the 8038 serves to establish a near-linear relationship between control voltage and output frequency (Tagliavini, 1975).

Because they yield a high and stable output voltage, two AND gates (Type MM74 C08) are interposed between $Q$ and $\bar{Q}$ of the F.F and the 5-Kohm potentiometers. The capacitor connected to Pins 10 and 11 of the 8038 determines the range of output frequency. There is an option of a wide variety of frequency bands, depending on the capacitance value selected (Mundl, 1981).

The lower part of Figure 1 shows the switching configuration that allows for individual adjustment of the amplitudes of both frequencies. The analog switch (National Semiconductor Corporation) is driven by the F-F and switches in such a way that one frequency at a time is applied to the summing amplifier. An example of a resulting waveform is shown at its output. Since the 8038 also generates square waves (Pin 9) and triangular waves (Pin 3), these waveforms may be used instead of the sine wave. Intermixing two different waveforms can be easily accomplished by separating the common input to the $10-\mathrm{Kohm}$ potentiometers (lower part of Figure 1).

As a whole, the generated pattern on the screen of the CRO can be shifted toward a higher or lower intensity by applying a dc voltage to an additional input arm of the summing amplifier. The transition points at which one frequency changes to another are dependent on the timing of the MVs and can, therefore, be set at any desired amplitude level. 


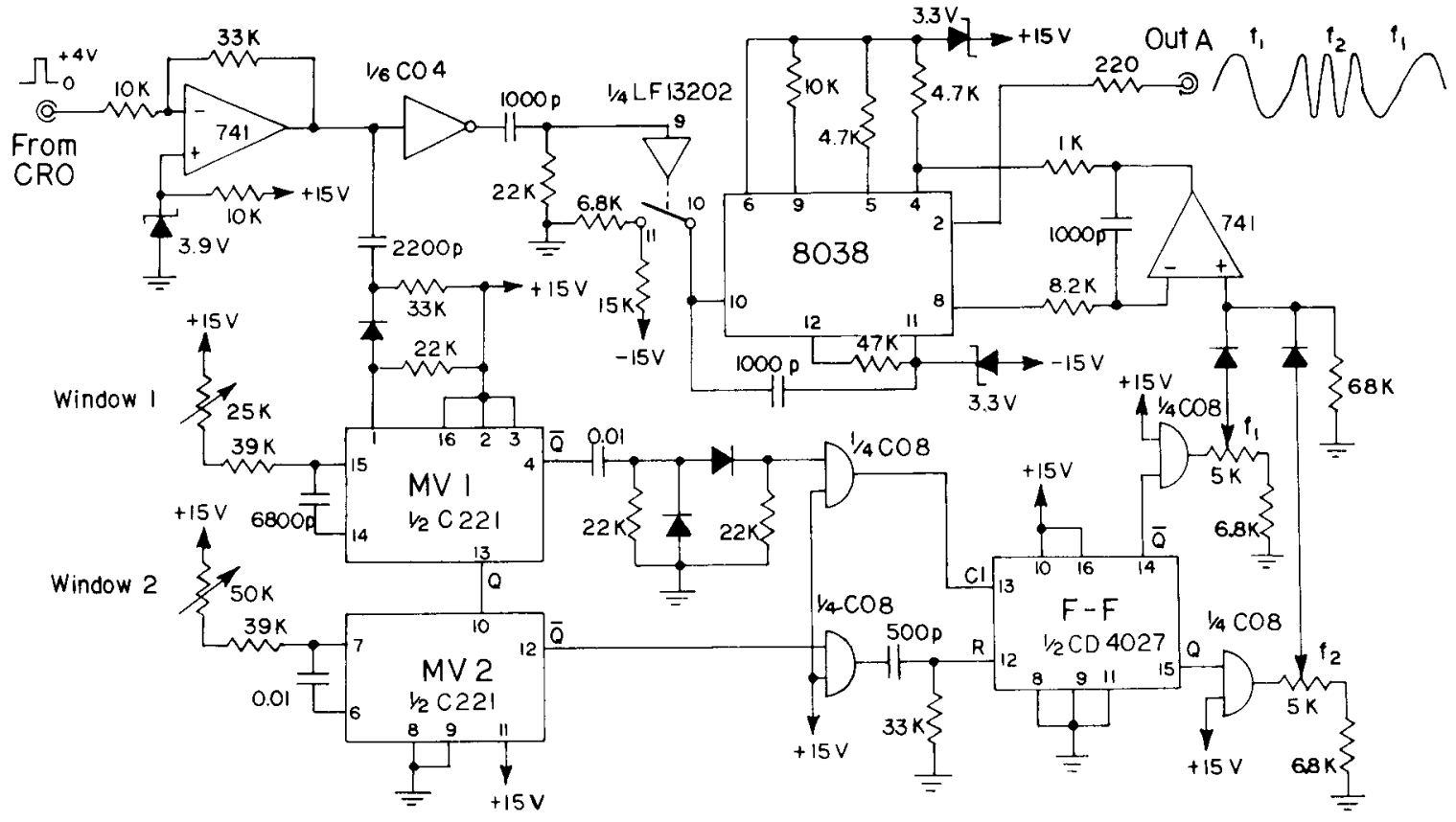

Analog Switch, LF 13202

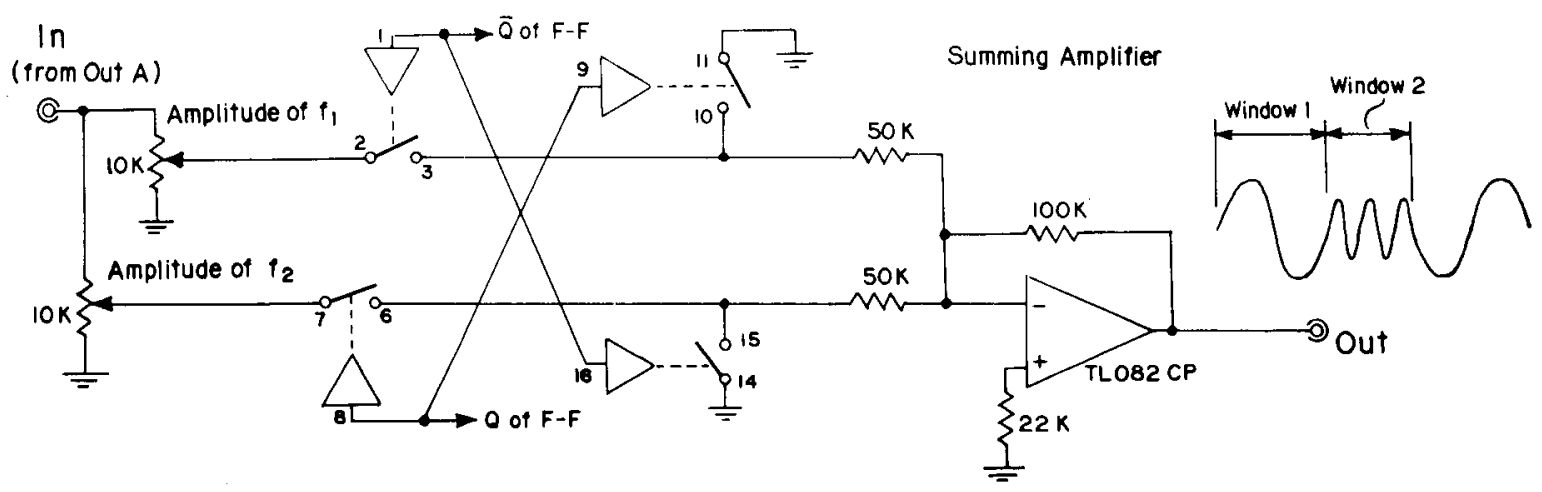

Figure 1. Schematic diagram. The Intersil 8038 function generator is programmed to produce sudden changes in frequency. The lower part of the figure shows a switching arrangement for adjusting the amplitude of each of the two frequencies. The circuit is powered by a regulated $\pm 15-\mathrm{V}$ supply.

\section{REFERENCES}

Mundl, W. J., \& Malmo, H. P. An ultrasonic auditory stimulator. Behavior Research Methods \& Instrumentation, 1981, 13, 328-330.
Tagliavini, A. Modified function generator yields linear VCO. Electronics, 1975, 48(22), 96-97.

(Received for publication December 23, 1981; accepted January 2, 1982.) 